\title{
Shunted-Josephson-junction model. II. The nonautonomous case
}

Belykh, V. N.; Pedersen, Niels Falsig; Sørensen, O. H.

\section{Published in:}

Physical Review B

Link to article, DOI:

10.1103/PhysRevB.16.4860

Publication date:

1977

Document Version

Publisher's PDF, also known as Version of record

Link back to DTU Orbit

Citation (APA):

Belykh, V. N., Pedersen, N. F., \& Sørensen, O. H. (1977). Shunted-Josephson-junction model. II. The nonautonomous case. Physical Review B, 16(11), 4860-4871. https://doi.org/10.1103/PhysRevB.16.4860

\section{General rights}

Copyright and moral rights for the publications made accessible in the public portal are retained by the authors and/or other copyright owners and it is a condition of accessing publications that users recognise and abide by the legal requirements associated with these rights.

- Users may download and print one copy of any publication from the public portal for the purpose of private study or research.

- You may not further distribute the material or use it for any profit-making activity or commercial gain

- You may freely distribute the URL identifying the publication in the public portal

If you believe that this document breaches copyright please contact us providing details, and we will remove access to the work immediately and investigate your claim. 


\title{
Shunted-Josephson-junction model. II. The nonautonomous case
}

\author{
V. N. Belykh* \\ Electronics Laboratory, The Technical University of Denmark, DK-2800 Lyngby, Denmark \\ N. F. Pedersen and O. H. Soerensen \\ Physics Laboratory I, The Technical University of Denmark, DK-2800 Lyngby, Denmark
}

(Received 26 October 1976)

\begin{abstract}
The shunted-Josephson-junction model with a monochromatic ac current drive is discussed employing the qualitative methods of the theory of nonlinear oscillations. As in the preceding paper dealing with the autonomous junction, the model includes a phase-dependent conductance and a shunt capacitance. The mathematical discussion makes use of the phase-space representation of the solutions to the differential equation. The behavior of the trajectories in phase space is described for different characteristic regions in parameter space and the associated features of the junction $I V$ curve to be expected are pointed out. The main objective is to provide a qualitative understanding of the junction behavior, to clarify which kinds of properties may be derived from the shunted-junction model, and to specify the relative arrangement of the important domains in the parameter-space decomposition.
\end{abstract}

\section{INTRODUCTION}

The great interest in the Josephson junction is to a large extent prompted by its far-reaching active and nonlinear behavior as a high-frequency element. Presently, a thorough basis for a theoretical understanding of the high-frequency properties of the device exists only for the Josephson tunnel junction. ${ }^{1}$ The basically phenomenological shunted-junction model is, however, able to account for a variety of experimental situations if the high-frequency excitation is incorporated in the form of a time-dependent bias current. This comparatively simple model, which apart from the nonlinear current contributions $I_{0} \sin \phi$ and $\epsilon G \cos \phi V$, contains only linear circuit elements, ${ }^{2}$ leads to a differential equation which cannot be solved analytically. We therefore think it worth while to undertake a detailed qualitative discussion of the model in order to point out, first, which kinds of properties are to be expected and, second, which field of parameters corresponds to a certain behavior.

We will keep the considerations on a qualitative level and present few quantitative results. The latter would require numerical or analog computations or approximate analytical methods, and is outside the scope of the present paper. Extensive work of this kind has been reported in the literature. Here we shall merely attempt to establish the basis for a deeper understanding of the shunted-junction model and provide a framework within which the behavior may be conveniently described.

Using the notation of Ref. 2, the outline of the present paper is as follows:
The theoretical considerations are contained in Sec. II. Here, the important bifurcations of the parameter space are presented in the $\rho, \beta$ plane for $|\epsilon|<1$. We have restricted the discussion to values of ac bias, $\alpha \lesssim 1$, since a discussion with no limitation on $\alpha$ would not be practicable. Also, the range $\alpha \lesssim 1$ is the more interesting from the point of view of small signal applications. The discussion is given in terms of the behavior of the phase-space trajectories corresponding to the different regions in the parameter space, and also the associated dc $I V$-curve features are described. Finally, estimates of the bifurcation curves are given. In Sec. III the results are further elucidated by reference to previously published results, and experimentally obtained $I V$-curves are used to illustrate some main points. Appendix A presents aspects of the mathematics of the phase-space pictures, and in Appendix B we state the criteria of stability of the periodic steady-state solutions.

\section{THEORY}

The equation governing the behavior of the shunted-junction model may in the nonautonomous case be written ${ }^{2}$

$$
\begin{aligned}
& \dot{\phi}=y, \\
& \dot{y}=\rho-\sin \phi-(1 / \sqrt{\beta})(1+\epsilon \cos \phi) y+\alpha \sin \Omega \tau,
\end{aligned}
$$

where $\rho=I_{\mathrm{dc}} / I_{0}, \alpha=I_{\mathrm{ac}} / I_{0}, \beta=2 e I_{0} C / \hbar G^{2}, \Omega=\omega / \omega_{0}$, and $\tau=\omega_{0} t$ with $\omega_{0}=\left(2 e I_{0} / \hbar C\right)^{1 / 2}$, and where the dot means differentiation with respect to normalized time.

If the parameter $\beta$ is small, a different normalization of time will be more convenient: $\tau_{1}=\omega_{c} t$, where $\omega_{c}=\omega_{0} \sqrt{\beta}=2 e I_{0} / \hbar G$, which with $\Omega_{1}=\omega / \omega_{c}$ 
result in the set of equations

$$
\begin{aligned}
& \dot{\phi}=y, \\
& \beta \dot{y}=\rho-\sin \phi-(1+\epsilon \cos \phi) y+\alpha \sin \Omega_{1} \tau_{1} .
\end{aligned}
$$

From the symmetry of Eqs. (1) and (2) it follows that only the range $\rho \geqslant 0$ needs consideration, since the system is invariant under the transformation

$$
\begin{aligned}
& \phi=-\tilde{\phi}, \quad y=-\tilde{y}, \quad \rho=-\tilde{\rho}, \quad \text { and } \\
& \Omega \tau=\Omega \tilde{\tau}+\pi, \quad \text { or } \quad \Omega_{1} \tau_{1}=\Omega_{1} \tilde{\tau}_{1}+\pi .
\end{aligned}
$$

Before going into a detailed discussion of the model behavior we shall derive a formal expression for the dc $I V$ curve. As mentioned in the preceding paper ${ }^{2}$ the $I V$ curve is determined by the steady-state solutions of Eqs. (1) or (2), i.e., by the equilibrium points and the periodic and almostperiodic trajectories in the phase space.

Assuming a periodic solution to Eq. (1): $\left(\phi^{*}, y^{*}\right)$ satisfying the condition

$$
\begin{aligned}
& \phi^{*}(\tau+2 \pi p / \Omega)=\phi^{*}(\tau)+2 \pi q, \\
& y^{*}(\tau+2 \pi p / \Omega)=y^{*}(\tau) ;
\end{aligned}
$$

i.e., with the time period $2 \pi p / \Omega[\phi(\bmod 2 \pi)]$, it follows from the Josephson voltage-phase relation expressed in normalized time that

$$
\begin{aligned}
V & =\frac{\hbar \omega_{0}}{2 e}\left\langle y^{*}\right\rangle \\
& =\frac{\hbar \omega_{0}}{2 e} \frac{\Omega}{2 \pi p}\left[\phi *\left(\tau+\frac{2 \pi p}{\Omega}\right)-\phi *(\tau)\right] \\
& =R \frac{\hbar \omega}{2 e},
\end{aligned}
$$

where $R=q / p$. Hence, any dc voltage determined by Eq. (5) with a rational value of $R$ corresponds to a periodic steady -state solution.

The Eq. (1) has only two kinds of steady-state solutions, that is, periodic and almost-periodic solutions. ${ }^{3}$ Furthermore, since $\dot{\phi} *=y^{*}$ is bounded ${ }^{4}$ $\left[\sqrt{\beta}(\rho-\alpha-1)<y^{*}<\sqrt{\beta}(\rho+\alpha+1)\right]$ for any steady state solution, $\phi^{*}$ cannot increase more rapidly than proportionally to $\tau$ as $\tau \rightarrow \infty$. Thus any steadystate solution satisfies

$$
\begin{aligned}
V & =\frac{\hbar \omega_{0}}{2 e} \lim _{\tau \rightarrow \infty} \frac{1}{\tau} \int_{0}^{\tau} \dot{\phi}^{*} d \tau \\
& =\frac{\hbar \omega_{0}}{2 e} \lim _{\tau \rightarrow \infty} \frac{\phi^{*}(\tau)}{\tau} \\
& =R \frac{\hbar \omega}{2 e},
\end{aligned}
$$

where $R$ here is some constant, which we shall denote the rotation character of the steady-state solution. Equation (6) includes Eq. (5) as a special case, and we conclude that if $R$ is rational $(R=q / p)$ then the solution is periodic in the sense of Eq. (4), whereas all dc voltages defined by irrational values of $R$ are determined by the almostperiodic solutions.

The general (implicit) expression for any part of the $I V$ curve corresponding to a certain value of $R$ is found by inserting the steady-state solution $\left(\phi_{R}^{*}\left(\tau_{\rho}\right), y_{R}^{*}\left(\tau_{\rho}\right)\right)$ into Eq. (1). Integration over the interval $\left(0, T_{\rho}\right)$ yields

$$
\begin{aligned}
y_{R}^{*}\left(T_{\rho}\right)-y_{R}^{*}(0)= & \int_{0}^{T} \rho\left(\rho-\sin \phi_{R}^{*}-\frac{1}{\sqrt{\beta}} y_{R}^{*}\right) d \tau_{\rho} \\
& +\frac{\epsilon}{\sqrt{\beta}}\left[\sin \phi *\left(T_{\rho}\right)-\sin \phi_{R}^{*}(0)\right] \\
& -\frac{\alpha}{\Omega}\left(\cos \Omega T_{\rho}-1\right) .
\end{aligned}
$$

The suffix $\rho$ on time variables is meant to indicate that time and $\rho$ may be functionally related. Dividing Eq. (7) by $T_{\rho}$ and substituting $T_{\rho}=2 \pi p / \Omega$ if the solution is periodic or letting $T_{\rho} \rightarrow \infty$ if the solution is almost periodic we obtain

$$
0=\rho-\left\langle\sin \phi_{R}^{*}\left(\tau_{\rho}\right)\right\rangle-(1 / \sqrt{\beta})\left\langle y_{R}^{*}\left(\tau_{\rho}\right)\right\rangle,
$$

or, expressed in terms of the junction voltage,

$$
\rho=\left\langle\sin \phi_{R}^{*}\left(\tau_{\rho}\right)\right\rangle+\left(G V / I_{0}\right)
$$

or, introducing the rotation character from Eq. (6),

$$
\rho=\left\langle\sin \phi_{R}^{*}\left(\tau_{\rho}\right)\right\rangle+R\left(\omega / \omega_{c}\right) 。
$$

The implicit Eqs. (8), (9), or (10) determine what we shall denote the $R$ branch of the dc $I V$ curve. Thus, in order to construct the complete $I V$ curve, we must for any fixed set of parameters $\rho, \beta, \epsilon, \alpha$, and $\Omega$ find all the steady-state solutions, periodic as well as almost periodic. Assuming for instance $\rho=\rho *$, we may obtain the set of solutions $R_{1}, R_{2}, \ldots, R_{n}$ each defining a certain branch of the $I V$ curve, where the stability of any specific branch under variation of $\rho$ may be determined using the methods described in Appendix B. Here we shall merely note that at any given moment of time the junction will be locked by one of the stable steady-state solutions selected by the initial conditions.

As in the automomous case $e^{2}$ this leads to the problem of parameter-space decomposition into domains, each corresponding to a certain set of steady-state solutions characterized by the set of values $R_{j}$. Below, a qualitative description of the parameter-space decomposition will be presented, and as we proceed the dependence of the $I V$ curve on the system parameters will be discussed. The pictorial representations of the trajectories in the phase space $G(\phi(\bmod 2 \pi), y, \tau(\bmod 2 \pi / \Omega))$ to be used 
in our general discussion are based on the Poincaré-Andronov point-mapping scheme outlined in some detail in Appendix A.

\section{A. Small $\beta$}

When the parameter $\beta$ is sufficiently small, we may use the theory of singular perturbed systems. ${ }^{5}$ If furthermore $|\epsilon|<1$, i.e., $1+\epsilon \cos \phi>0$, Eq. (2) has the integral surface $y=I\left(\phi, \tau_{1}, \beta\right)$ where only the dependence on parameter $\beta$ has been explicitly displayed. The integral surface is periodic in $\phi$ and $\tau_{1}$ and asymptotically stable in the whole phase space $G$. This means that any steady-state solution to Eq. (2) belongs to the surface $y=I\left(\phi, \tau_{1}, \beta\right)$, i.e., satisfies the equation

$$
\dot{\phi}=I\left(\phi, \tau_{1}, \beta\right) \text {. }
$$

As the function $I\left(\phi, \tau_{1}, \beta\right)$ is periodic in $\phi$ with period $2 \pi$ and in $\tau_{1}$ with period $T_{1}=2 \pi / \Omega_{1}$ the integral surface may be pictured as the torus: $\left(\phi(\bmod 2 \pi), \tau_{1}\left(\bmod 2 \pi / \Omega_{1}\right)\right)$. According to the theory of systems with a toroidal integral surface ${ }^{6}$ the rotation number $r$ defined by

$$
r=\lim _{\tau_{1} \rightarrow \infty} \frac{\phi\left(\tau_{1}, \phi_{0}, \beta\right)}{\tau_{1}}
$$

is a characteristic quantity of Eq. (11). In Eq. (12) $\phi\left(\tau_{1}, \phi_{0}, \beta\right)$ is a solution to (11) with the initial condition $\phi_{0}=\phi\left(0, \phi_{0}, \beta\right)$. Comparing the definition of the rotation number (12) to Eq. (6), it follows that the rotation number and the rotation character are identical for solutions on the torus. We will nevertheless maintain the distinction because on the torus the rotation number has specific properties $^{6}$ which are essential for the understanding of the $I V$-curve behavior:

(i) The rotation number $r$ is a unique and continuous function of the system parameters. This implies that the $I V$ curve is single valued and without discontinuous voltage jumps.

(ii) If the rotation number is rational, $r=q / p$, then and only then Eq. (11) has the periodic steadystate solution, $\phi_{r}^{*}\left(\tau_{1}\right)$, satisfying the condition

$$
\dot{\varphi}_{r}^{*}\left(\tau_{1}+p T_{1}\right)=\phi_{r}^{*}\left(\tau_{1}\right)+2 \pi q .
$$

If the rotation number is irrational, then and only then Eq. (11) has the almost-periodic steady-state solution generating the whole torus surface, $y$ $=I\left(\phi, \tau_{1}, \beta\right)$ in $G$. These properties justify the identification between the rotation character $R$ and the rotation number $r$ defined for solutions on the torus.

(iii) If and only if the rotation number is rational, $r=q / p$, and the periodic function

$$
g\left(\phi_{0}\right)=\phi^{*}\left(p T_{1}, \phi_{0}, \beta\right)-2 \pi q-\phi_{0}
$$

changes sign in the interval $\phi_{0} \in(0,2 \pi]$ the rotation number is stable under variance of the function $I\left(\phi, \tau_{1}, \beta\right)$. If the initial condition, $\phi_{0}$ $=\phi^{*}\left(0, \phi_{0}, \beta\right)$ satisfies $g\left(\phi_{0}\right)=0$, the solution is the periodic solution $\phi_{r}^{*}\left(\tau_{1}, \phi_{0}, \beta\right)$ with rotation number $r$.

The important consequence of property (iii) is that the voltage $V$ determined by Eq. (5) may stay constant while $\rho$ is varied only if the rotation number is rational. In other words, rf-induced constant-voltage steps may occur only at voltages $V=(q / p)(\hbar \omega / 2 e)$. The stability of the corresponding steady-state periodic solution $\varphi_{r}^{*}\left(\tau_{1}, \phi_{0}, \beta\right)$ is reflected by the change of sign of $g\left(\phi_{0}\right)$ in $\phi_{0}$ $\in(0,2 \pi]$. The smooth parts of the $I V$ curve connecting the end points of the constant-voltage steps are determined both by the unstable steadystate periodic solutions (with rational $r$ ) satisfying $g\left(\phi_{0}\right) \equiv 0$ for any $\phi_{0}$ and by the steady-state almostperiodic solutions (with irrational $r$ ) which are always unstable.

In the limit $\beta \ll 1$ the function $I\left(\phi, \tau_{1}, \beta\right)$ may be written in the form

$$
I\left(\phi, \tau_{1}, \beta\right)=I\left(\varphi, \tau_{1}, 0\right)+f\left(\varphi, \tau_{1}, \beta\right),
$$

where

$$
I\left(\varphi, \tau_{1}, 0\right)=\left(\rho-\sin \varphi+\alpha \sin \Omega_{1} \tau_{1}\right) /(1+\epsilon \cos \varphi)
$$

and

$$
\lim _{\beta \rightarrow 0} f\left(\phi, \tau_{1}, \beta\right)=0 .
$$

Accordingly, deviations between the $I V$ curve of the system (11) and the $I V$ curve of the system.

$$
\dot{\phi}=I\left(\phi, \tau_{1}, 0\right)
$$

may at most be of order $\beta$. This means that any large current steps (large of order $\beta$ ) at constant voltage must be common to both systems, whereas small additional current steps (of order $\beta$ or less) may appear in the $I V$ curve of system (11) as $\beta$ is increased from zero. Keeping in mind that such small modifications are possible, we shall continue the discussion of the $I V$-curve behavior in the small- $\beta$ limit based on some specific properties of the rotation number of the system (16).

(iv) The rotation number of the system (16) is a nondecreasing function of the parameter $\rho$. This is seen from Eqs. (15) and (16) showing that the angular velocity $\dot{\phi}$ for fixed $\phi$ and $\tau_{1}$ is an increasing function of $\rho$. Thus, $r \propto V \propto\langle\dot{\phi}\rangle$ cannot decrease and the $I V$ curve, $V=V(\rho)$, must be a nondecreasing function of $\rho$, possibly constant in some regions of $\rho$ where $r$ is rational.

(v) In the interval

$$
0<\rho<1-\alpha
$$


the rotation number is $r=0$ and stable.

Since the function $I\left(\phi, \tau_{1}, 0\right)$ in Eq. (16) for $0<\rho$ $<1-\alpha$ and any $\tau_{1}$ satisfies the inequalities

$$
\begin{aligned}
& I\left(-\frac{1}{2} \pi, \tau_{1}, 0\right)>0, \\
& I\left(\frac{1}{2} \pi, \tau_{1}, 0\right)<0,
\end{aligned}
$$

the two solutions $\phi_{1}\left(\tau_{1},-\frac{1}{2} \pi\right)$ and $\varphi_{2}\left(\tau_{1}, \frac{1}{2} \pi\right)$ to Eq. (16) with the initial conditions $\phi_{0}=-\frac{1}{2} \pi$ and $\varphi_{0}=\frac{1}{2} \pi$, respectively, satisfy the inequalities

$$
-\frac{1}{2} \pi<\phi_{1}<\frac{1}{2} \pi \text {, and } \frac{1}{2} \pi>\phi_{2}>-\frac{1}{2} \pi
$$

for any $\tau_{1} \in\left(0, T_{1}\right]$. This implies that the function (14) with $p=1$ and $q=0$ satisfies

$$
g\left(-\frac{1}{2} \pi\right)>0 \text { and } \quad g\left(\frac{1}{2} \pi\right)<0 .
$$

Consequently, according to property (iii) of the rotation number we must have $r=0$ and stable in the interval (17). The maximum zero-voltage current, $\rho_{\alpha}$, must be greater than $1-\alpha$ and due to Eq. (10) also less than 1:

$$
0<1-\alpha<\rho_{\alpha}<1 \text {. }
$$

In the two limiting cases, $\Omega_{1} \ll 1$ and $\Omega_{1} \gg 1$, the estimate of $\rho_{\alpha}$ may be expressed differently. As a consequence of the results in Sec. II C we have

$$
\begin{aligned}
& \rho_{\alpha}=1-\alpha+O\left(\Omega_{1}\right), \quad \Omega_{1} \ll 1 \\
& \rho_{\alpha}=1-O\left(\Omega_{1}^{-1}\right), \quad \Omega_{1} \gg 1
\end{aligned}
$$

where $O(x)$ is some function of $x$ such that $\lim _{x \rightarrow 0} O(x)=0$ as $x$ or faster.

For one specific value of $\alpha$ and any $\Omega_{1}<1$ we can give an upper limit on $\rho_{\alpha}$ which is more restrictive than Eq. (21). For $\rho=\Omega_{1}$ and $\alpha=\left[1+\left(\epsilon \Omega_{1}\right)^{2}\right]^{1 / 2} \mathrm{Eq}$. (16) has the solution

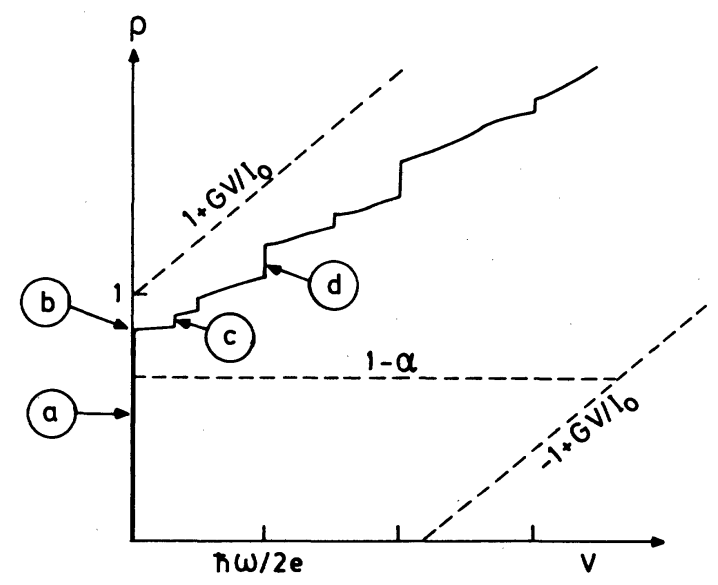

FIG. 1. Qualitative behavior of the $I V$ curve in the nonhysteretic small- $\beta$ limit. The figure shows that constant-voltage steps may be present at voltages $(q / p)(\hbar \omega / 2 e)$. The encircled letters show the points or regions of existence of the solutions shown in Fig. 2.
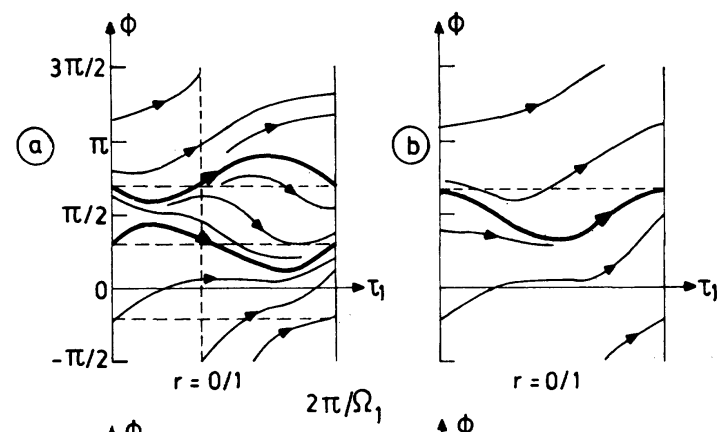

(c)

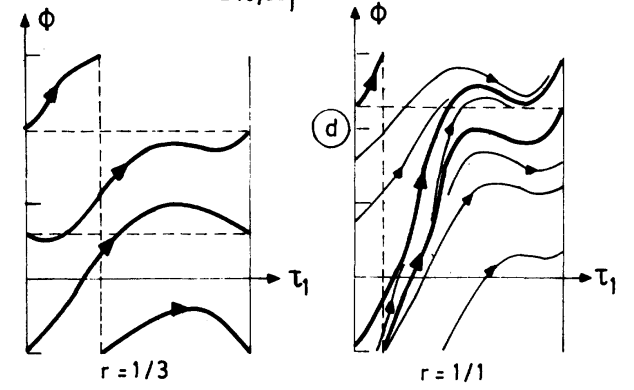

FIG. 2. Time evolution of the phase in the small- $\beta$ limit. The individual pictures are marked with letters corresponding to the arrows in Fig. 1.

$$
\varphi=\Omega_{1} \tau_{1}-\tan ^{-1}\left(\epsilon \Omega_{1}\right), \quad y=\Omega_{1} .
$$

This solution defines a point on the $r=1$ branch of the $I V$ curve. Then by necessity the upper limit of the $r=0$ branch $\rho_{\alpha}$ must be less than $\Omega_{1}$.

$$
\rho_{\alpha}<\Omega_{1} \text { for } \alpha=\left[1+\left(\epsilon \Omega_{1}\right)^{2}\right]^{1 / 2} \text {. }
$$

On the basis of this discussion the $I V$ curve shown in Fig. 1 may be drawn. The figure shows qualitatively the features to be expected. The maximum zero-voltage current has been suppressed but satisfies the inequalities (21). The junction voltage is a single-valued and nondecreasing function of $\rho$. For some rational values of the rotation number at voltages given by Eq. (5), constant-voltage current steps have appeared reflecting the locking by a periodic stable steadystate solution. Also indicated in the figure is the general result that the $I V$ curve displacements must be within limits

$$
-1+G V / I_{0}<\rho(V)<1+G V / I_{0}
$$

which is a consequence of Eq. (9).

Qualitative pictures of the trajectories on the torus are shown in Figs. 2(a), 2(b), 2(c) and 2(d). The steady-state solutions are shown as heavy lines whereas the thin lines show solutions which asymptotically approach the steady-state solutions. In no case does more than one stable steady-state solution exist. In Fig. 2(a), we have one stable and one unstable oscillating solution 


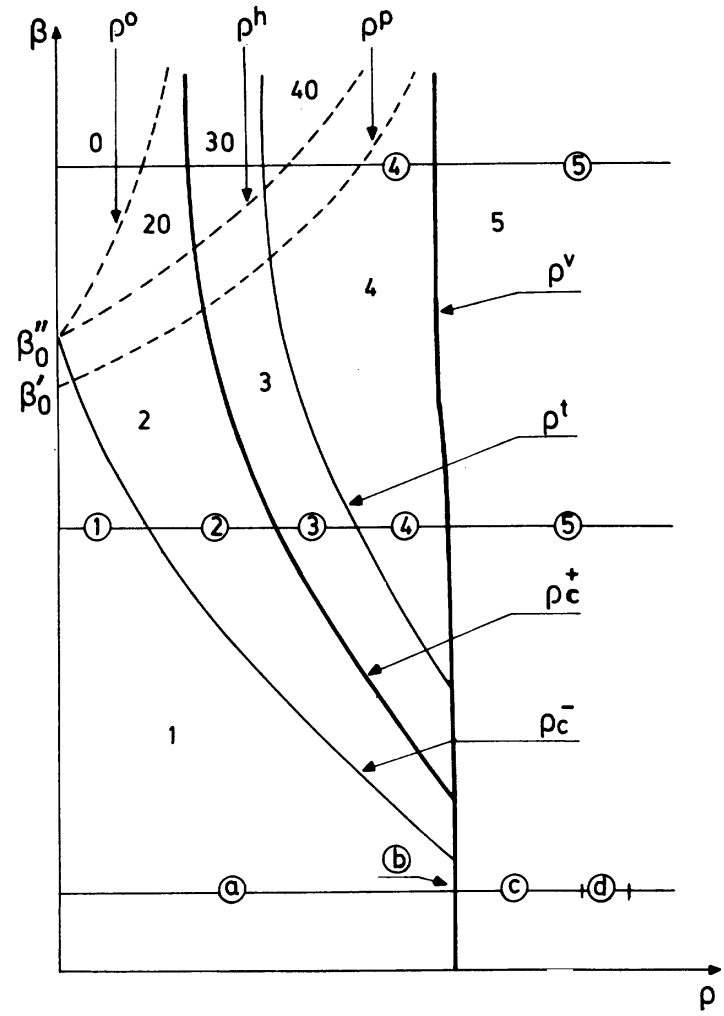

FIG. 3. Important bifurcations of the parameter space shown in the $\rho, \beta$ plane. The curves are only qualitative, valid for $\alpha \lesssim \frac{1}{2}$ and $|\epsilon|<1$. Three representative values of $\beta$ are chosen (the horizontal lines) and the junction behavior as a function of $\rho$ for these values of $\beta$ is discussed in the text.

(O-type) with $q=0$, which in Fig. 2(b) have merged into one. In Fig. $2(\mathrm{c})$ is shown a rotating solution ( $r$-type) with $q=1$ and $p=3$, and Fig. 2(d) shows the stable and the unstable $r=1 / 1 r$-type solutions.

\section{B. General case}

Below a more general discussion valid for any $\beta$ will be given. In order to keep the discussion on a manageable level only the parameter regions $|\epsilon|<1, \alpha \lesssim 1$, and any positive $\beta, \rho$, and $\Omega$ will be considered. Some consequences of having $\epsilon>1$ will, however, be briefly discussed. The decomposition of the parameter space into regions of qualitatively different behavior is a convenient starting point. The decomposition is more complicated than was the case for the autonomous junction ${ }^{2}$ because of the larger number of parameters involved. From each characteristic region typical phase-space portraits of the junction state will be drawn. Rather than attempting to draw the three-dimensional pictures in the phase space $G$ : $(\phi, y, \tau)$ we will use the Poincare-Andronov point- mapping operator $L$ of the cylinder $(\phi, y)$ into itself as described in Appendix A.

The most significant bifurcation curves decomposing the parameter space for constant $\epsilon, \Omega$, and $\alpha<\frac{1}{2}$ are shown qualitatively in Fig. 3 in the $\rho, \beta$ plane. ${ }^{4}$ The behavior of the separatrix invariant curves and the closed (periodic in $\phi$ ) invariant curves of the point mapping $L$ characteristic of the different domains of the decomposition are sketched in Fig. $4 .^{4}$

The lower horizontal line in Fig. 3 corresponds to the small- $\beta$ limit discussed in Sec. II A. In this case the point mapping $L$ has the unique stable and closed invariant curve generated by intersecting the integral surface $y=I(\phi, \tau, \beta)$ with the plane, $\tau=$ const [this curve bears a strong resemblance to the rotating-limit cycle of the autonomous case and is shown in Fig. 4(5)]. The invariant curve, being defined by the equation $y$ $=I(\phi, \tau, \beta)$, has the fixed points corresponding to the periodic solutions on the torus [cf. Fig. 2(d)]. The letters on the lower horizontal line in Fig. 3 indicate the points and regions of existence of the solutions shown in Fig. 2.

For intermediate values of $\beta$ we follow the center horizontal line in Fig. 3. A variation of $\rho$ along this line produces the sequence of phase portraits shown in Figs. 4(1),4(2), .., 4(5). For small $\rho$ [region 1, Fig. 4(1)], the junction is locked by the unique $O$-type solution with $R=0(q / p=0 / 1)$ attracting the whole phase space. This solution generates the fixed point $M^{+}$. Figure 4 is drawn for a value of $\beta$ where the fixed point is of the focal or spiral type, which is different from the point-mapping representation of Fig. 2(a). On the torus the separatrix invariant curves would join smoothly forming a node-type fixed point rather than the spiral fixed point in
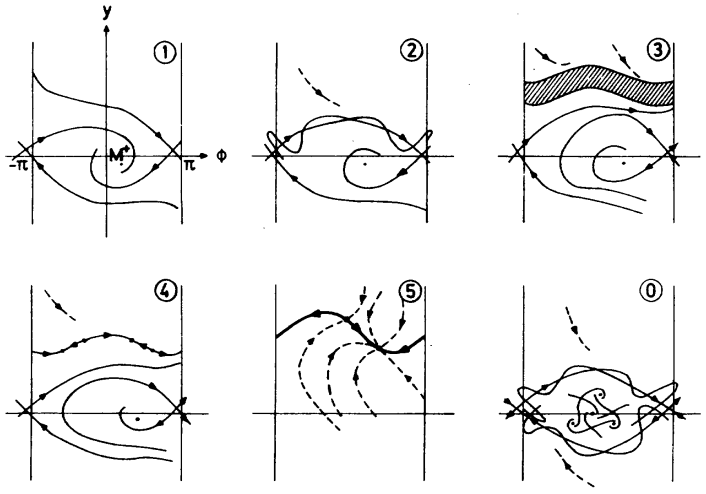

FIG. 4. Phase-plane portraits in the point-mapping representation of the junction states at the points or regions marked with corresponding numbers in Fig. 3. 
Fig. 4(1). The bifurcation curve separating these two distinct types of behavior is not shown in Fig. 3.

An increase of $\rho$ for fixed $\beta$ towards the curve $\rho=\rho_{c}^{-}(\beta, \epsilon, \alpha, \Omega)$ creates irregularities in the upper separatrix invariant curves such that at $\rho_{c}^{-}$ they intersect tangentially at an infinite number of points. A further increase of $\rho$ into region 2 creates the transverse intersections shown in Fig. 4(2), and an infinite number of $r$-type unstable solutions are generated. Due to the intersections the system has the structural stable homoclinic rotating curves. ${ }^{4,7}$ Increasing $\rho$ across the boundary $\rho_{c}^{+}$into region 3 causes the intersections to disappear but instead appears the ring [the hatched region in Fig. 4(3)] girding the cylinder. The ring attracts those trajectories which are not attracted by the fixed point $M^{+}$. The behavior of the solutions inside the ring changes at least at an infinite number of $\rho$ values ${ }^{7,8}$ as $\rho$ is increased through region 3 . The behavior is complicated and little is known of the details. However, as $\rho$ approaches the curve $\rho^{t}$ an infinite number. of stable $r$-type periodic solutions exist, some weakly stable, and others strongly stable, under variation of $\rho$. Inside region 3 the system may jump unpredictably between the various $\boldsymbol{r}$-type solutions and we suggest the term "rotation scattering" to describe the situation. As $\rho$ reaches the curve $\rho^{t}$ the behavior becomes calm, and beyond $\rho=\rho^{t}$ the stable unique periodic invariant curve has appeared and continues to exist for every $\rho>\rho^{t}$ [Fig. 4(4)]. At the curve $\rho=\rho^{V}$ the stable and unstable $O$-type solutions merge and disappear when the curve is crossed. Hence, in region 5 the system has only the stable periodic invariant curve, and the curve $\rho^{V}$ defines the maximum zero-voltage current, $\rho_{\alpha}=\rho^{V}$, for intermediate values of $\beta$. In region 5 the steady-state solutions are confined to the torus. The invariant curve in Fig. 4(5) is drawn for a specific value, $r=1 / 1$, of the rotation number, and the two fixed points shown are generated by the stable and the unstable trajectories shown in Fig. 2(d).

When $\rho$ is increasing from zero, the junction will stay locked by the $O$-type solution $M^{+}$until the bifurcation curve $\rho=\rho^{V}$ is reached. Passing $\rho=\rho^{V}$ the fixed point $M^{+}$disappears and the junction jumps to a finite-voltage state. In the region $\rho>\rho^{V}$ the steady-state $r$-type solution is on the torus and the $I V$ curve will be qualitatively similar to the small $\beta$ case, i.e., the voltage is a nondecreasing single-valued function of $\rho$ with constant voltage steps at some rational values of the rotation number.

For decreasing $\rho$ starting in region 5 the junction stays locked by the steady-state trajectories on the torus until the curve $\rho=\rho^{t}$ is reached. Thus, in region 4 the $I V$ curve has two branches, one being the zero-voltage branch, the other determined by the rotation number on the torus. In region 3: $\rho_{c}^{+}<\rho<\rho^{t}$, we have in addition to the $V=0$ solution a large number of stable solutions with $V \neq 0$. The observed $I V$ curve depends on which of these solutions locks the junction, and on the region of stability of that particular solution. The $\boldsymbol{r}$-type stable solutions in region 3 are confined inside the ring in Fig. 4(3). We may have several strongly stable solutions within the ring and also an infinite number of weakly stable and unstable ones. The strongly stable solutions may have regions of stability overlapping in $\rho$ such that the constant voltage steps can be hysteretic if $\rho$ is varied within the boundaries of region 3 . The junction voltage may jump discontinuously between values $R_{1} \hbar \omega / 2 e, R_{2} \hbar \omega / 2 e, \ldots$, where $R_{n}$ is some rational number, or may follow a smooth curve consisting of an infinite number of infinitely small steps corresponding to the periodic and almost-periodic solutions with vanishing regions of attraction. The junction cannot enter the zerovoltage state in region 3 . That will, however, happen at some point in region 2: $\rho_{c}^{-}<\rho<\rho_{c}^{+}$. When $\rho$ is decreasing from $\rho_{c}^{+}$towards $\rho_{c}^{-}$the probability for having experienced a voltage jump to $V=0$ increases to certainty. Region 2 and 3 are both regions of very complicated behavior ${ }^{7,8}$; only in region 2 is the zero-voltage branch included among the accessible final states of the "scattering" process. The jump back to zero vol tage may happen anywhere in region 2 but must happen for some $\rho \geqslant \rho_{c}^{-}$. The qualitative behavior of the $I V$ curve for intermediate values of $\beta$ is summarized in Fig. 5, where the extent of the different regions $1,2,3,4$, and 5 is indicated on the $\rho$ axis.

Finally, the junction behavior for large values of $\beta$ is illustrated following the upper horizontal line in Fig. 3. At $\rho=0$ and everywhere in region 0 (separated from region 20 by the curve $\rho^{0}$ ) the separatrix invariant curves have transverse intersections as shown in Fig. 4(0). Due to these intersections the system has an infinite number of unstable solutions of the $O$-type as well as of the $r$ type (both with positive and negative values of $R$ ) and may have also some stable solutions of these types. With initial conditions $\rho=0, y_{0}=0$ the junction may be locked by one of the stable $O$-type periodic solutions $(q=0, p>1)$ which exist in the large $\beta$ region above the curve $\rho^{p}$ in Fig. 3. The point-mapping picture of such a solution (with $q$ $=0, p=3)$ is shown in Fig. 4(0). A variation of $\rho$ inside region 0 may produce a different $0 / p$ solution replacing the original one. This may happen without noticeable change of the $I V$ curve. In- 


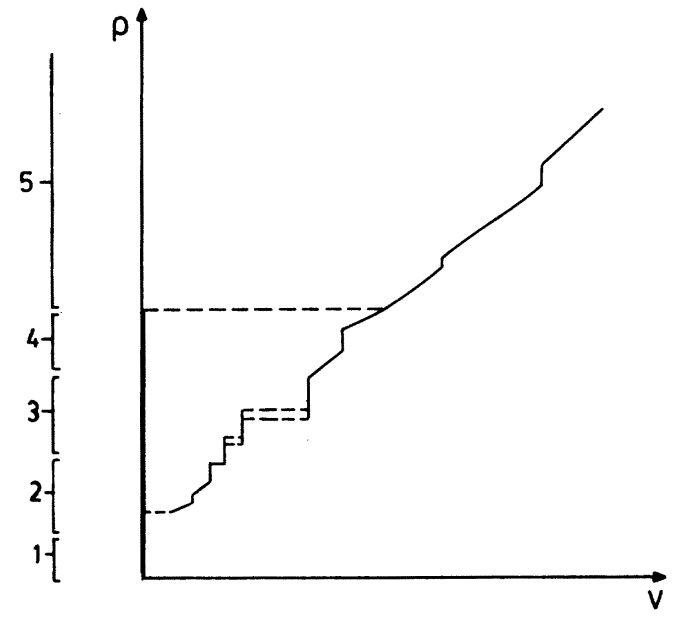

FIG. 5. Qualitative behavior of the $I V$ curve in the intermediate $\beta$ case. On the $\rho$ axis is indicated the extent of the regions $1,2, \ldots, 5$ of the parameter space in Fig. 3.

creasing $\rho$ we arrive at the curve $\rho^{0}$, where the intersections between the lower separatrix invariant curves become tangential and a further increment of $\rho$ into region 20 causes these intersections to disappear. The point-mapping picture for region 20 is like that for region 2 [Fig. 4(2)] except that additional $O$-type solutions $(q=0, p>1)$ exist, and also the left-hand-side upper and lower separatrix invariant curves have transverse intersections. Similarly, the pictures for the regions 30 and 40 resemble those for regions 3 and 4 . It should be noted, however, that the intersections between the left-hand-side upper and lower separatrix invariant curves do not disappear until the curve $\rho^{h}$ is crossed, and for $\rho>\rho^{p}$, only the fundamental $(q=0, p=1) O$-type solution remains. For $\rho$ above $\rho^{h}$ or $\rho^{t}$, depending on which is larger, the $r$-type solutions are on the torus and the junction behavior is simple.

The $I V$ curve will be similar to the intermediate $\beta$ case with the following exceptions:

(i) For increasing $\rho$ switching from $V=0$ to a finite voltage may occur for $\rho<\rho^{V}$. This phenomenon is caused by the intersections between the left-hand-side upper and lower separatrix invariant curves. This makes a "random scattering process" possible which takes the junction state from an $O$-type solution with $\dot{V}=0$ to a stable $r$ type solution with $V \neq 0$ via a number of closely spaced intermediate states. The jump to finite voltage may occur anywhere in the region: $\rho_{c}^{+}$ $<\rho<\rho^{h}$. If, however, the junction has not switched at $\rho=\rho^{h}$ it will stay on the $V=0$ branch up to $\rho=\rho^{V}$.

(ii) The point of return to $V=0$ will be some- where: $\rho<\rho_{c}^{+}$.

(iii) The existence of the different $O$-type solutions with $q=0, p>1$ is not reflected in the $I V$ curve, but will be observable via the associated changes in the junction frequency spectrum as $\rho$ is varied on the zero-voltage branch.

So far we have described the qualitative behavior of the shunted-junction model for $|\epsilon|<1$ on basis of Fig. 3 which is valid for $\alpha \lesssim \frac{1}{2}$. For larger values of $\alpha$ the bifurcation curve $\rho=\rho^{V}$ will tend to zero implying that all $O$-type solutions disappear. Some part of region 3 may, however, expand into region $5\left(\rho^{t}>\rho^{v}\right.$ in some range of $\left.\beta\right)$. The picture shown in Fig. 4(3) does still apply for region 3 except that the fixed point $M^{+}$does not exist. The observable consequence of the spreading of region 3 is that the $R$ branches of the $I V$ curve may be hysteretic also for $\rho>\rho^{V}$.

Finally, a brief comment on the case $\epsilon>1$. The bifurcation curves, $\rho^{0}, \rho^{h}$, and $\rho^{p}$ move upwards on the $\beta$ axis as $\epsilon$ is increased above 1. For $\alpha$ $<1$ and intermediate values of $\beta$ the curve $\rho_{c}^{-}$ splits into two. This splitting is correlated to the similar splitting of the curve $\rho_{c}(\beta, \epsilon)$ reported in the autonomous case. ${ }^{2}$ For increasing $\rho$, a stable closed invariant curve is generated at the crossing of the additional bifurcation curve $\rho=\rho_{d}^{\prime}$ giving rise to a finite-voltage solution. The only significant difference between the $I V$ curves for $|\epsilon|<1$ and $\epsilon>1$ is observed when $\rho$ is decreased while the junction is biased at finite voltages. If $\epsilon>1$, the jump back to zero voltage occurs at the curve $\rho=\rho_{a}^{\prime}$ where the stable closed invariant curve loses its stability.

\section{Approximations for the bifurcations}

For sufficiently small $\Omega(\Omega \ll 1)$ the bifurcation curves $\rho_{c}^{+}, \rho_{c}^{-}, \rho^{0}$, and $\rho^{V}$ may be written in the form ${ }^{5}$

$$
\begin{aligned}
& \rho_{c}^{+}=\rho_{c}(\beta, \epsilon)+\alpha-O(\Omega), \\
& \rho_{c}^{-}=\rho_{c}(\beta, \epsilon)-\alpha+O(\Omega), \\
& \rho^{0}=-\rho_{c}(\beta, \epsilon)+\alpha-O(\Omega) ; \beta>\beta_{0}^{\prime \prime}, \\
& \rho^{V}=1-\alpha+O(\Omega),
\end{aligned}
$$

where $\beta_{0}^{\prime \prime}$ is defined by $\rho^{0}\left(\beta_{0}^{\prime \prime}\right)=0$ and $\rho_{c}(\beta, \epsilon)$ is the bifurcation curve of the autonomous system, which to a good approximation may be written ${ }^{2}$

$$
\rho_{c}=(4 / \pi \sqrt{\beta})\left(1+\frac{1}{3} \epsilon\right) \text {. }
$$

The stable $O$-type solution is in the quasistatic approximation

$$
\phi *(\tau)=\sin ^{-1}(\rho+\alpha \sin \Omega \tau) .
$$

For large values of $\Omega(\Omega \gg 1)$ Eq. (1) may, with renormalized time scale $\tau_{2}=\Omega \tau$, be written 
$\dot{\phi}=\Omega^{-1} y$,

$\dot{y}=\Omega^{-1}\left[\rho-\sin \phi-(1 / \sqrt{\beta})(1+\epsilon \cos \phi) y+\alpha \sin \tau_{2}\right]$.

Using the Krylov-Bogoliubov theorem ${ }^{3}$ for the explicit averaging in time $\tau_{2}$ we obtain from Eq. (30) the autonomous system, the solutions of which approximate the solutions of $(30)$ in the time interval $0<\tau_{2}<\Omega$. Hence, when $\Omega \gg 1$ we have

$$
\begin{aligned}
& \rho_{c}^{+}=\rho_{c}(\beta, \epsilon)+O\left(\Omega^{-1}\right), \\
& \rho_{c}^{-}=\rho_{c}(\beta, \epsilon)-O\left(\Omega^{-1}\right), \\
& \rho^{V}=1-O\left(\Omega^{-1}\right), \\
& \beta_{0}^{\prime}=O(\Omega), \quad\left[\text { where } \rho^{p}\left(\beta_{0}^{\prime}\right)=0\right] .
\end{aligned}
$$

Note, that for large $\Omega$ the regions 2 and 3 are compressed into the neighborhood of the autonomous bifurcation curve $\rho_{c}$ and that $\beta_{0}^{\prime}$ is large of order $\Omega$. This means that the switching point from finite to zero voltage occurs always very close to $\rho=\rho_{c}$. For decreasing $\Omega$ the regions 2 and 3 expand and $\beta_{0}^{\prime}$ moves down into the intermediate $\beta$ range. From Eq. (27) we obtain the inequalities defining the boundaries of region 2 , and hence, the $\rho$ interval in which the return to the zero-voltage branch occurs

$$
\rho_{c}-\alpha<\rho_{c}^{-}<\rho_{c}^{+}<\rho_{c}+\alpha .
$$

As far as the curve $\rho^{t}$ is concerned we merely state that the region 3 is narrow if $\alpha$ is small, i.e.,

$$
\rho^{t}=\rho_{c}^{+}+O(\alpha) \text {. }
$$

An estimate of the bifurcation curve $\rho=\rho^{p}$ which separates the regions 20,30 , and 40 from the regions 2,3 , and 4 may be given on basis of Ref. 9 . The curve $\rho=\rho^{p}$ runs in the large $\beta$ region

$$
\beta>\left[1-(\rho-\alpha)^{2}\right]^{-1 / 2} ; \rho<1-\alpha
$$

which with $\rho=0$ gives the lower limit of the point $\beta_{0}^{\prime}$

$$
\beta_{0}^{\prime}>\left(1-\alpha^{2}\right)^{-1 / 2} .
$$

In the case, $\epsilon>1$, an additional bifurcation curve $\rho_{d}^{\prime}$ appears below the curve $\rho=\rho_{c}^{-}$and is correlated to the curve $\rho=\rho_{d}$ where ${ }^{2}$

$$
\rho_{d}=[\epsilon(2 \beta-\epsilon)]^{1 / 2} / \beta \text {. }
$$

The curve $\rho=\rho_{d}^{\prime}$ runs somewhere within the band

$$
\rho_{d}-\alpha<\rho_{d}^{\prime}<\rho_{d}+\alpha \text {. }
$$

When $\alpha$ is sufficiently small all the bifurcation curves $\rho_{c}^{-}, \rho_{c}^{+}$, and $\rho^{t}$ approach the curve $\rho_{c}$ and $\rho^{V}$ is close to 1 . The $I V$ curve will then be similar to the $I V$ curve in the autonomous case, except that small current steps (of order $\alpha$ ) may have appeared.

\section{DISCUSSION AND CONCLUSION}

In this section we shall further illustrate the results of the qualitative considerations presented in Sec. II and their implications for the various ways of behavior to be expected. We must keep in mind, however, that the shunted-junction model on which everything here is based is phenomenological and that a real junction may have properties which cannot be accounted for within the model. One phenomenon, which is clearly not included in the shunted-junction model, is the Dayem-Wyatt effect, ${ }^{10}$ i.e., the enhancement of the zero-voltage current step under the influence of microwave radiation of ten observed for superconducting weak links. This follows from Eq. (9) showing that the normalized maximum zero-voltage current $\rho_{\alpha}$ may never exceed $\rho=1$, the value obtained for $\alpha=0$.

The most striking modification of the $I V$ curve when a time-dependent excitation is present is the appearance of the constant voltage steps, which are very well accounted for within the shunted-junction model. In mathematical terms these current steps reflect the finite region of stability of a periodic steady-state solution [periodic in the sense of Eq. (4)] under variation of the parameter $\rho$. It is well established from experiments and from analog computations that the step heights $\Delta \rho$ oscillate as the rf amplitude $\alpha$ is varied. The periodic solution on a particular step (with $R=q / p$ ) must make $q$ rotations of $2 \pi$ in $\phi$ in each period $2 \pi p / \Omega$ of time independent of $\alpha$. What distinguishes the behavior of the solution on the different lobes of the $\Delta \rho$ vs $\alpha$ pattern is that the $q$ full rotations are the net result of a rotation $2 \pi q^{+}$ in the positive direction followed by a rotation $2 \pi q^{-}$in the negative direction such that $q^{+}-q^{-}$ $=q$, and the number of full rotations in either direction increases by 1 going from one lobe to the next.

In general we cannot say which steps are stable and which are not without going through the stability analysis described in Appendix B for each periodic solution defined by a rational rotation character $R=q / p$. There is one exception. If we restrict ourselves to the range $\alpha<1$ the solution with rotation character, $R=0$ (which generate the zero-voltage current), is always stable for $\rho<$ $1-\alpha$. From Eqs. (27) and (31) it further follows that the maximum zero-voltage current depends on $\alpha$ as

$$
\begin{aligned}
& \rho_{\alpha} \simeq 1-\alpha ; \quad \Omega \ll 1 \\
& \rho_{\alpha} \lesssim 1 ; \quad \Omega \gg 1
\end{aligned}
$$

for very low and very high frequencies, respectively. The low-frequency result is in agreement 


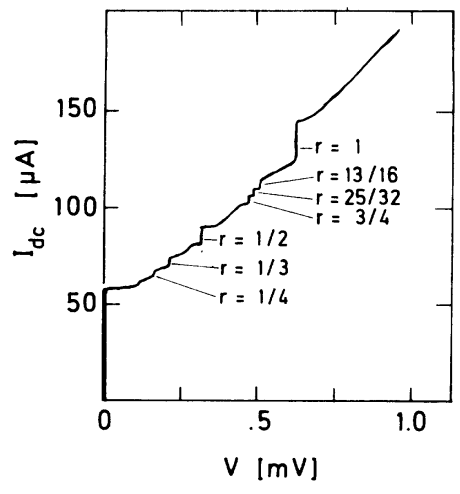

FIG. 6. Experimental $I V$ curve for a $\mathrm{Nb}-\mathrm{Nb}$ point contact at $T=4.2 \mathrm{~K}$ irradiated by $295-\mathrm{GHz}$ microwaves.

with analog computer results ${ }^{11}$ and an approximately linear dependence on $\alpha$ has also been obtained analytically in the low $\beta$ limit. ${ }^{12}$ In the high-frequency limit the junction behaves as if voltage biased, implying that $\rho_{\alpha}$ will oscillate with $\alpha$ as a zero-order Bessel function. Hence, the first term in the expansion of the function $O\left(\Omega^{-1}\right)$ in Eq. (31) is $O\left(\Omega^{-1}\right)=\beta \alpha^{2} / 2 \Omega^{2}+\cdots$.

One important result from the applications point of view is that the junction in the small- $\beta$ limit will be nonhysteretic $\left[\beta<\beta_{1}\right.$, where $\left.\rho_{c}^{-}\left(\beta_{1}\right)=1\right]$. This follows from the theorem of the uniqueness of the rotation number for solutions on the torus. As above, the stability region of any rational rotation number must be evaluated using Appendix B. Analog computations have shown ${ }^{13}$ that for $\beta$ $=0$ and $\epsilon=0$ only steps with integer values of $r$ may have nonzero regions of stability and that the fractional or subharmonic steps appear as $\beta$ is increased from zero. An analytical treatment of the case $\beta=0, \epsilon=0$ have shown ${ }^{12}$ that the subharmonic steps do not exist; in contradiction to this, others ${ }^{14}$ have by numerical calculations found subharmonic steps present also for $\beta=0$ and $\epsilon=0$. We cannot resolve this discrepancy. If $\epsilon \neq 0$, the existence of subharmonic steps does not per se violate any mathermatical theorems. Figure 6 shows an $I V$ curve obtained for a $\mathrm{Nb}-\mathrm{Nb}$ point contact irradiated by $300-\mathrm{GHz}$ microwaves. This junction is in the nonhysteretic regime and shows a rich selection of subharmonic steps classified by their rotation number.

For intermediate values of $\beta$ the $I V$ curve is also nonhysteretic for values of dc bias $\rho$ in excess of $\rho^{t}(\beta, \epsilon, \alpha, \Omega)$. This is illustrated in Fig. 7 showing the $N$ curve for a Sn-SnO-Sn tunnel junction in an $X$-band microwave field. The figure corresponds to the case mentioned in Sec. II where we have region 3 reaching across $\rho=\rho^{V}$. into region 5. When that happens (for values of $\alpha \gtrless \frac{1}{2}$ ) we may have hysteretic steps for values of $\rho>\rho^{V}$. The step $r=3$ in Fig. 7 is, however, without hysteresis. The figure also illustrates that inside the regions of complicated behavior, stable solutions exist with overlapping regions of stability.

If $|\epsilon|<1$ in the intermediate $\beta$ range the voltage jump from finite to zero voltage occurs for decreasing $\rho$ at some point inside region $2 ; \rho_{c}^{-}<\rho$ $<\rho_{c}^{+}$. The actual switching point cannot be predicted theoretically due to the complicated structure of the steady-state solutions. This does not mean, however, that the $I V$ curve is necessarily irreproducible at this point, particularly if precautions have been made to eliminate random perturbations of the system (such as noise and power-line transients). The consequence is simply that the coexistence of many solutions with different rotation characters opens the possibility that the $I V$ curve changes drastically following a minor change of parameters. If $\epsilon>1$, the situation is different. The complicated regions 2 and 3 still exist, but the jump back to the zero-voltage state is well-defined in the sense that the value of $\rho=\rho_{d}^{\prime}$ where it happens may be calculated numerically. As in the autonomous case ${ }^{2}$ the jump to zero voltage for decreasing $\rho$ is distinctly different depending on whether $|\epsilon|<1$ or $\epsilon>1$. It will, however, be difficult to design an experiment which unambiguously excludes other explanations.

The most prominent feature in the large $\beta$ region is the existence of the $O$-type solutions with $q=0$ and $p>1$. We cannot state precisely the regions of existence of a specific $O$-type solution without resorting to numerical calculations, but

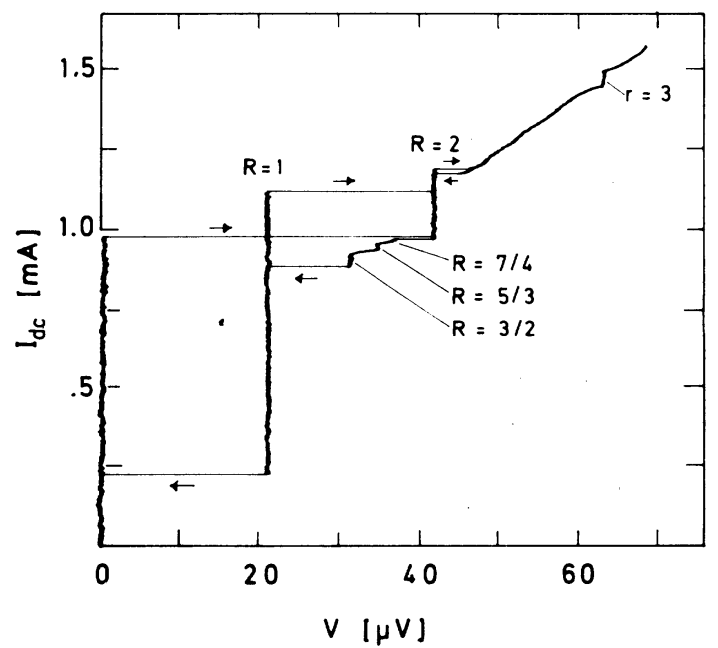

FIG. 7. Experimental $I V$ curve for a Sn-SnO-Sn tunnel junction at $T=3.69 \mathrm{~K}$ irradiated by $9.9-\mathrm{GHz}$ microwaves. The arrows show the direction of current sweep. 


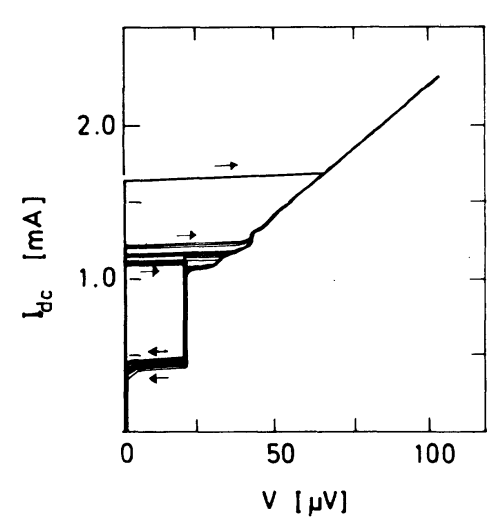

FIG. 8. Experimental $I V$ curve for a Sn-SnO-Sn tunnel junction at $T=3.60 \mathrm{~K}$ irradiated by $9.9-\mathrm{GHz}$ microwaves. The $I V$ curve has been retraced several times in the direction indicated by the arrows.

just note that a bifurcation curve $\rho=\rho^{p}(\beta, \epsilon, \alpha, \Omega)$ exists such that these subharmonic oscillations are present only for values of $\beta$ above this curve. As mentioned in Sec. II, the subharmonic oscillations are not directly evident in the dc $I V$ curve. If, however, the frequency of the subharmonic oscillation, $\Omega / p$, at some value of $\rho$ coincides with an internal resonance, e.g., the Josephson plasma resonance with frequency $\left(1-\rho^{2}\right)^{1 / 4}$, the amplitude may blow up and the properties of the dc $I V$ curve are modified. ${ }^{15}$ The excitation of the plasma frequency has also been observed (for $p=2)^{16}$ by direct measurement of the subharmonic amplitude.

If $\beta$ is above the curve $\rho^{h}(\beta, \epsilon, \alpha, \Omega)$ in Fig. 3 a characteristic behavior may be observed. For values of $\rho \in\left(\rho_{c}^{+}, \rho^{h}\right)$ the zero-voltage branch becomes unstable and a transition to finite voltages is likely to occur anywhere in this interval. The return to zero voltage may happen anywhere in the region $\rho<\rho_{c}^{+}$. This phenomenon is illustrated in Fig. 8 , which shows the $I V$ curve for a $\mathrm{Sn}-\mathrm{SnO}-\mathrm{Sn}$ tunnel junction (the same as in Fig. 7 but at a lower temperature). The $I V$ curve has been retraced several times without change of external parameters, and the figure demonstrates that the jump to finite voltages may happen either within a fairly broad band of bias currents or at a specific much larger value of $\rho\left(\rho=\rho^{V}\right)$. The point of return to zero voltage is also not well defined.

In order to utilize the (parametric) generation of subharmonic oscillations, the crucial problem is to obtain an efficient coupling to the input-output lines, i.e., to match their impedance to the junction. This problem becomes increasingly difficult to solve as the working frequency is raised, due to the dominating shunt capacitance of the large $\beta$ junction. This difficulty is further emphazised in the light of Eq. (31) showing that the region of subharmonic oscillations moves towards larger $\beta$ 's as the frequency is raised $\left[\beta_{0}^{\prime}\right.$ $=O(\Omega)]$.

Within the limitations of the shunted-Josephsonjunction model, we hope in this paper to have established the basis for a deeper qualitative understanding of the Josephson-junction behavior under the influence of a monochromatic ac bias. We have pointed out the more significant bifurcations, and classified the different regions with respect to the associated junction properties. Even though few quantitative results have been presented, we believe our discussion to be useful in providing some guidelines for prediction of the junction behavior in the various regions of parameter space. Finally, it deserves mentioning that many of the qualitative results are more general than explicitly stated in this paper. The mathematical theorems used here do also apply to systems with nonsinusoidal current-phase relations.

\section{ACKNOWLEDGMENTS}

One of use (V. N. B.) wishes to thank Professor G. Bruun and the Electronics Laboratory, The Technical University of Denmark for their hospitality during his stay.

\section{APPENDIX A: THE POINT MAPPING}

The nonautonomous system (1) can be considered as a third-order system if the equation $\dot{\tau}=1$ is added. On the other hand, the nonautonomous system (1) being periodic in time is equivalent to a point mapping ${ }^{8}$ of the cylinder $(\phi, y)$ into itself. This is understood as follows:

Any solution to the system (1) $\phi\left(\tau, \phi_{0}, y_{0}, \tau_{0}\right)$, $y\left(\tau, \phi_{0}, y_{0}, \tau_{0}\right)$ with the initial conditions $\phi_{0}=\phi\left(\tau_{0}, \phi_{0}, y_{0}, \tau_{0}\right), y_{0}=y\left(\tau_{0}, \phi_{0}, y_{0}, \tau_{0}\right)$ satisfies, because of the periodicity in $\tau$,

$$
\begin{aligned}
& \phi\left(\tau, \phi_{0}, y_{0}, \tau_{0}\right)=\phi\left(\tau+2 \pi / \Omega, \phi_{0}, y_{0}, \tau_{0}+2 \pi / \Omega\right), \\
& y\left(\tau, \phi_{0}, y_{0}, \tau_{0}\right)=y\left(\tau+2 \pi / \Omega, \phi_{0}, y_{0}, \tau_{0}+2 \pi / \Omega\right),
\end{aligned}
$$

for any $\tau, \phi_{0}, y_{0}$, and $\tau_{0}$. However, Eq. (A1) implies that $\tau$ can be considered as $\tau(\bmod 2 \pi / \Omega)$. Choosing the cylinder $\tau=\tau_{0}$ in the phase space $G$ (equivalent to any cylinder $\tau=\tau_{0}+2 \pi k / \Omega$; $k$ integer), the solution to Eq. (1) generates a point mapping of the cylinder into itself. The initial point $M_{0}$ : $\left(\phi_{0}, y_{0}\right)$ at $\tau=\tau_{0}$ is transferred into the point $M_{1}$ : $\left(\dot{\phi}_{1}, y_{1}\right)$ at $\tau_{1}=\tau_{0}+2 \pi / \Omega$, where

$$
\left.\begin{array}{l}
\phi_{1}=\phi\left(\tau_{1}, \phi_{0}, y_{0}, \tau_{0}\right) \\
y_{1}=y\left(\tau_{1}, \phi_{0}, y_{0}, \tau_{0}\right)
\end{array}\right\} \text { or } M_{1}=L M_{0} \text {. }
$$

Since $\tau_{1}=\tau_{0}(\bmod 2 \pi / \Omega)$, the step may be repeated 
and bring us from $M_{1}$ at $\tau=\tau_{0}$ to $M_{2}:\left(\phi_{2}, y_{2}\right)$ at $\tau_{2}$ $=\tau_{0}+2 \pi / \Omega=\tau_{0}(\bmod 2 \pi / \Omega)$ which is the next point on the cylinder $\tau=\tau_{0}$. The point mapping $L:\left(\phi, y, \tau_{0}\right)$ $\rightarrow\left(\phi, y, \tau_{0}\right)$ defined by Eq. (A2) is called the Poincare-Andronov operator and is widely used in the theory of nonlinear oscillations.

There exists an obvious correspondance between the periodic solutions to Eq. (1) and the fixed points of the mapping $L$. Let, e.g., the system have the periodic solution $\left(\phi^{*}(\tau), y^{*}(\tau)\right)$ with

$\phi *(\tau+p T)=\phi *(\tau)+2 \pi q, \quad y^{*}(\tau+p T)=y *(\tau)$,

where $T=2 \pi / \Omega$. This solution is of the oscillating type ( $O$-type) if $q=0$ and of the rotating type $(r-$ type) if $R=q / p \neq 0$. Then the point-mapping $L$ has the fixed point $M_{q}=\left(\phi^{*}\left(\tau_{0}\right)+2 \pi q, y^{*}\left(\tau_{0}\right)\right)$ in the sense

$$
L^{p} M_{0}=M_{q}, \quad\left[M_{q}(\phi \bmod 2 \pi)=M_{0}\right],
$$

where Eq. (A4) defines the $O$-type discrete cycle $M_{0}, L M_{0}, L^{2} M_{0}, \ldots, L^{p} M_{0}=M_{0}$ if $q=0$ and the $r$-type discrete cycle $M_{0}, L M_{0}, L^{2} M_{0}, \ldots, L^{p} M_{0}=M_{q}$ if $q \neq 0$.

Furthermore, the integral surfaces of the system (1) are in a one-to-one correspondance to the invariant curves of $L$, where the curve $\Gamma: F(\phi, y)$ $=0$ is an invariant curve of $L$ if for any point $M$ $\in \Gamma, L M \in \Gamma$.

For the interpretation of the Josephson-junction behavior two types of invariant curves of $L$ are important: the saddle fixed point separatrices, and the closed invariant curves periodic in $\phi$. On the cylinder $\left(\phi, y, \tau_{0}\right)$ these curves look like the separatrices and limit cycles of the autonomous system $^{2}$ except for one essential difference. In the autonomous case, the point $(\phi, y)$ moves continuously in time along the separatrices or limit cycles, whereas in the nonautonomous case, the point moves in discrete steps along the corresponding invariant curves.

The separatrix invariant curves of one and the same saddle fixed point may have transverse intersections as illustrated in Figs. 4(2) and 4(0). These intersections generate discrete trajectories of $L$ doubly asymptotic to the saddle fixed point and are called the homoclinic curves. ${ }^{4,7}$ In the neighborhood of the intersections, we have complicated structures containing an infinite number of periodic stable and unstable trajectories. For a detailed description of the complicated structures and their dependence on the parameters of the point mapping the reader is referred to the mathematical literature. ${ }^{4,7}$

\section{APPENDIX B: EXISTENCE AND STABILITY OF PERIODIC SOLUTIONS}

Let us assume that Eq. (1) has the steady-state solution $\Lambda_{R}(\rho)=\left(\phi_{R}^{*}(\tau, \rho), y_{R}^{*}(\tau, \rho)\right)$ with period $2 \pi p /$
$\Omega$, i.e., periodic in the sense of Eq. (4). The solution may be determined numerically using the iteration procedures for the point mapping ${ }^{17} \mathrm{de}-$ fined by Eq. (A2). Obviously, the magnitude of the current step for a certain rational value of $R$ is determined by the stability region $\rho_{1}<\rho<\rho_{2}$ of the solution $\Lambda_{R}(\rho)$. Thus the boundaries $\rho_{1}$ and $\rho_{2}$ are determined as the points where the stability is lost. Below, we state the stability criteria for a particular solution.

Inserting

$$
\phi=\phi_{R}^{*}(\tau, \rho)+\xi, \quad y=y_{R}^{*}(\tau, \rho)+\eta
$$

into Eq. (1) and expanding to first order in $(\xi, \eta)$ we obtain the linear time-dependent equation periodic in time with period $2 \pi p / \Omega$

$$
\dot{\xi}=\eta, \quad \dot{\eta}=-\gamma(\tau, \rho) \xi-\lambda(\tau, \rho) \eta
$$

with

$$
\begin{aligned}
\gamma(\tau, \rho)= & \cos \phi_{R}^{*}(\tau, \rho) \\
& -(\epsilon / \sqrt{\beta}) \sin \phi_{R}^{*}(\tau, \rho) y_{R}^{*}(\tau, \rho)
\end{aligned}
$$

and

$$
\lambda(\tau, \rho)=(1 / \sqrt{\beta})\left[1+\epsilon \cos \phi_{R}^{*}(\tau, \rho)\right] .
$$

The trivial solution $\xi=\eta=0$ to Eq. (B2) corresponds to the solution $\Lambda_{R}(\rho)$ of Eq. (1). Thus the stability of $\Lambda_{R}(\rho)$ is determined by the stability of the trivial solution to Eq. (B2).

The criteria of asymptotic stability ${ }^{18}$ of the periodic steady-state solution $\Lambda_{R}(\rho)$ are as follows:

If the two roots $\mu_{1}$ and $\mu_{2}$ of the characteristic equation for the linear system (B2) at time $\tau_{0}$ $=2 \pi p / \Omega$ are inside the unit circle in the complex plane, then the solution $\Lambda_{R}(\rho)$ is asymptotically stable. ${ }^{19}$

The stability is lost if either one or both of the roots reach the unit circle. When $\mu_{1}$ reaches the value +1 the solution $\Lambda_{R}(\rho)$ merges with the saddle-type unstable solution. The solution becomes unstable and disappears. This happens at the bifurcation curve $\rho=\rho^{V}$ in Fig. 3 and at bifurcation curves for the rotation number $(R=r)$ on the torus. Also when $\mu_{1}$ reaches the value -1 , the solution becomes unstable and disappears (in this case an unstable periodic solution with period $4 \pi p / \Omega$ is generated $\left.^{8}\right)$. This behavior may be observed inside region 3 [(Fig. 3 and Fig. 4(3)]. Finally, when $\mu_{1,2}= \pm e^{i \theta}$ the periodic solution $\Lambda_{R}(\rho)$ becomes unstable but continues to exist for $\left|\mu_{1,2}\right|>1$ as an unstable solution. Here, various subharmonic oscillations are generated (with periods greater than $2 \pi p / \Omega$ ) depending on the argument $\theta$. This type of behavior may be observed at the bifurcation curve $\rho=\rho^{p}$, and inside the regions 2,3 , 20,30 , and 40 . 
*Permanent address: Scientific-Research Institute of Applied Mathematics and Cybernetics, Gor'kii University, Gor'kii, USSR.

${ }^{1}$ N. R. Werthamer, Phys. Rev. 147, 255 (1966); L. E. Hasselberg, M. T. Levinsen, and M. R. Samuelsen, J. Low Temp. Phys. 21, 567 (1975); R. E. Harris, Phys. Rev. B 13, 3818 (1976).

${ }^{2}$ V. N. Belykh, N. F. Pedersen, and O. H. Soerensen, Phys. Rev. B 16, 4853 (1977), preceding paper.

${ }^{3}$ N. N. Bogoliubov and Yu. A. Mitropolsky, Asymptotic Methods in the Theory of Non-Linear Oscillations (Gordon and Breach, New York, 1962).

${ }^{4}$ L. N. Belynstina and V. N. Belykh, Diff. Eq. $\underline{9}, 309$ (1973); ibid. 9, 453 (1973); Phase Synchronization, edited by L. $\bar{N}$. Belynstina and V. V. Shakhgil'djan (Svyaz, Moscow, 1975), Chap. 6 (in Russian).

${ }^{5} \mathrm{Yu}$. A. Mitropolsky and O. V. Lykova, Integral Manifolds in Nonlinear Mechanics (Nauka, Moscow, 1973) (in Russian).

${ }^{6}$ V. A. Pliss, Nonlocal Problems of the Theory of Oscillations (Academic, New York, 1966).

${ }^{7}$ S. Smale, Bull. Am. Math. Soc. 73,747 (1967); N. K. Gavrilov and L. P. Shil'nikov, Math. of the USSR, Sbornik 17, 467 (1972); ibid. 19, 139 (1973).

${ }^{8} \mathrm{Yu}$. I. Neimark, The Method of Point-Mapping in the Theory of Nonlinear Oscillations (Nauka, Moscow, 1972) (in Russian).
${ }^{9}$ V. N. Belykh, Differ. Uravn. 11, 1738 (1975) (in Russian).

${ }^{10}$ A. F. G. Wyatt, V. M. Dmitriev, W. S. More, and F. W. Sheard, Phys. Rev. Lett. 16, 1166 (1966); A. H. Dayem and J. J. Wiegand, Phys. Rev. 155, 419 (1967).

${ }^{11}$ P. Russer, Acta Phys. (Austriaca) 32, 374 (1970); H. Fack and V. Kose, J. Appl. Phys. 42, 320 (1971). ${ }^{12}$ M. J. Renne and D. Polder, Rev. Phys. Appl. $\underline{9}, 25$ (1974).

${ }^{13} \mathrm{C}$. A. Hamilton and E. G. Johnson, Jr., Phys. Lett. A 41,393 (1972).

${ }^{14} \mathrm{~J} . \mathrm{R}$. Waldram, A. B. Pippard, and J. Clarke, Philos. Trans. R. Soc. Lond. A 268, 265 (1970).

${ }^{15}$ C. K. Bak, B. Kofoed, N. F. Pedersen, and K. Saermark, J. Appl. Phys. 46, 886 (1975); IEEE Trans. Magn. MAG-11, 829 (1975).

${ }^{16} \mathrm{~J}$. Mygind, N. F. Pedersen, and O. H. Soerensen, Appl. Phys. Lett. 29, 317 (1976).

${ }^{17}$ T. J. Aprille and T. N. Trick, Proc. IEEE 60, 108 (1972).

${ }^{18} \mathrm{E}$. A. Coddington and N. Levinson, Theory of Ordinary Differential Equations (McGraw-Hill, New York, 1955), Chaps. 3, 13.

${ }^{19}$ In the point-mapping formulation the roots $\mu_{1}$ and $\mu_{2}$ are the eigenvalues of the Jacobian of the pointmapping operator $L$ taken at the point $\phi=\phi_{R}^{*}\left(\tau_{0}, \rho\right)$, $y=y_{R}^{*}\left(\tau_{0}, \rho\right)$. 